\title{
Inventario de gases efecto invernadero en la Universidad de Bogotá Jorge Tadeo Lozano (Utadeo)
}

\section{Inventory of greenhouse gases at the Universidad de Bogotá Jorge Tadeo Lozano (Utadeo)}

\author{
Daniela Manso Piñeros \\ Departamento de Ciencias Biológicas y Ambientales, Facultad de Ciencias Naturales e Ingeniería, \\ Universidad de Bogotá Jorge Tadeo Lozano, Bogotá, D. C., Colombia
}

Carmen Alicia Parrado Moreno

Departamento de Ciencias Biológicas y Ambientales, Facultad de Ciencias Naturales e Ingeniería,

Universidad de Bogotá Jorge Tadeo Lozano, Bogotá, D. C., Colombia

http://orcid.org/0000-0002-2013-8491

Andrés Julián Aristizábal

Departamento de Ingeniería, Facultad de Ciencias Naturales e Ingeniería, Universidad de Bogotá Jorge Tadeo Lozano, Bogotá, D. C., Colombia

http://orcid.org/0000-0002-7571-2913

andresj.aristizabalc@utadeo.edu.co

Fecha de recepción: 29 de junio de 2017 Fecha de aceptación: 10 de octubre de 2017

Sugerencia de citación: Manso Piñeros, D., Parrado Moreno, C. A. y Aristizábal, A. J. (2017). Inventario de gases efecto invernadero en la Universidad de Bogotá Jorge Tadeo Lozano (Utadeo). Mutis, 7(2), 44-58. doi: http://dx.doi.org/10.21789/22561498.1252

Editor: Hernández Fernández, J. javier.hernandez@utadeo.edu.co

\section{RESUMEN}

Las universidades, como instituciones de educación superior, deben asumir compromisos ambientales, y estos compromisos requieren políticas claras y acertadas, así como la adopción de estrategias que involucren la cuantificación de sus impactos. En este estudio se presenta el inventario de gases de efecto invernadero (GEI) en la Universidad de Bogotá Jorge Tadeo Lozano (Utadeo), sede Bogotá, correspondiente al año 2015. Mediante la identificación y clasificación de las principales actividades desarrolladas en la institución y la posterior colección de datos de entrada de materia y energía se cuantificaron las emisiones de $\mathrm{GEI}$ en $\mathrm{CO}_{2}$ equivalentes, acorde con la norma Iso 14064-1 (2006). En los límites organizacionales de la Utadeo se establecieron diez fuentes de emisión. En el alcance I se calcularon $138.163 \mathrm{t} \mathrm{CO}_{2} \mathrm{e}$, atribuido a procesos de combustión de diésel y gas natural en el funcionamiento de plantas eléctricas, cafeterías y laboratorios; en el alcance $\mathrm{II}, 628.833 \mathrm{t} \mathrm{CO}_{2} \mathrm{e}$, que corresponden a las emisiones indirectas por consumo de energía eléctrica en diversas actividades educativas y académicas. Además, en emisiones indirectas del alcance III se cuantificaron $921.368 \mathrm{t} \mathrm{CO}_{2} \mathrm{e}$, asociadas a la infraestructura, el transporte aéreo, consumo de papel, agua y generación de residuos. El inventario de GEI de la Universidad fue de $1688.36 \mathrm{t} \mathrm{CO}_{2} \mathrm{e}$, y las emisiones comprendidas en el alcance III fueron las que hicieron mayor aporte.

Palabras clave: gases efecto invernadero, alcances I, II y III, impacto, consumo y energía. 


\section{ABSTRACT}

In this study we estimated the emissions of Greenhouse Gases of the Universidad de Bogotá Jorge Tadeo Lozano, Bogota, for 2015; using the international methodology ISo 14064-1 by means of the identification and classification of the main activities that are developed in the institution and the subsequent quantification of the amount of $\mathrm{CO}_{2}$ e emitted associated with them. It was possible to establish within the organizational limits of the university ten sources of emission. In the direct emissions of scope I, a total of $138.16 \mathrm{t} \mathrm{co}_{2}$ e was obtained, attributed to processes of combustion of diesel and natural gas in the operation of power plants, coffee shops and laboratories, followed by scope II with $628.83 \mathrm{t} \mathrm{CO}_{2} \mathrm{e}$, which correspond to the indirect emissions by consumption of electric energy in the development of various educational and academic activities and in the other indirect emissions the scope III with a total of $921.382 \mathrm{t} \mathrm{cO}_{2} \mathrm{e}$, mainly in construction, air transport, paper consumption, water and waste generation activities. The total emission of GHG from the University was $1688.3 \mathrm{t} \mathrm{CO}_{2} \mathrm{e}$, with the scope III being the largest amount of GHG being generated within the university.

Keywords: Greenhouse gases, scope I, II and III, impact, consumption and energy.

\section{INTRODUCCIÓN}

El sistema climático de la Tierra se caracteriza por variar a lo largo del tiempo, con alternancia en las condiciones climáticas desde que la atmósfera evolucionó hasta su estado actual como consecuencia de procesos naturales y humanos. Por ejemplo, en los últimos 500 millones de años se presentaron cuatro períodos muy cálidos y cuatro períodos fríos o de fríos extremos, relacionados específicamente con el forzamiento orbital (Hannah, 2015). Sin embargo, en las últimas décadas se han evidenciado cambios, no solo a causa de factores naturales, sino también por el desarrollo de actividades antrópicas de tipo industrial y comercial que responden al modelo de consumo y producción presente (Mapama, 2017). El impacto atmosférico por GEl relacionados con la actividad humana ha dado lugar al forzamiento radiativo del sistema climático, lo que modifica el efecto de la radiación de la energía del sol, el calentamiento de la atmósfera y produce incertidumbre climática (Hannah, 2015).

Hoy en día, los fenómenos asociados al cambio climático son cada vez más comunes, dado que los eventos ligados a este ocurren con mayor frecuencia con impactos sobre el nivel del mar, los glaciares y los bosques, entre otros. La principal causa de este fenómeno es el incremento en la concentración de gases efecto invernadero (GEI), que se acumulan en la atmósfera y aumentan la temperatura media de la Tierra y, por tanto, alteran el ciclo natural del sistema climático del planeta (National Aeronautics and Space Administration, 2017). Por ejemplo, las emisiones de $\mathrm{CO}_{2}$ se consideran la causa principal del cambio climático (Parga y Romero, 2013) y fueron responsables de cerca del $10 \%$ del aumento del forzamiento radiativo entre el 2005 y 2012 (Myhre et al., 2013). Además, en los países industrializados representan aproximadamente el $80 \%$ de las emisiones de GEI, destinadas a permanecer en la atmósfera entre cincuenta y doscientos años, dependiendo de cómo se reciclen en la tierra o en los océanos (Comisión Europea, 2003).

El aumento de la temperatura evidencia transformaciones únicas en la historia. En primer lugar, la rapidez con la que estas variaciones están teniendo lugar en períodos de tiempo cortos respecto a la evolución del planeta; en segundo lugar, el hecho de que una única especie, el Homo sapiens, es el motor de todos los cambios (Crutzen y Stoermer, 2000), lo que constituye no solo un problema ambiental, sino también de desarrollo con impactos potenciales en la sociedad, la economía y los ecosistemas (Duarte, Alonso, Benito, Dachs, Montes, Pardo Buendía y Valladares, 2006). Por lo anterior, el Panel Intergubernamental sobre Cambio Climático (IPCC) y diversas instituciones 
trabajan en la elaboración de guías, protocolos, directrices y metodologías internacionales que permitan obtener indicadores a partir de la cuantificación de las emisiones y aportes al calentamiento. Entre estos se encuentra el inventario de GEI de organizaciones estandarizado por la Iso 14064 (2006), que detalla los principios y requisitos para diseñar, desarrollar, administrar e informar inventarios de GEI a nivel organizacional. Este indicador permite cuantificar los gases emitidos de manera directa e indirecta por una institución. El inventario parte de una cuantificación de las entradas de materia y energía, para luego ser multiplicadas por un factor de emisión; de este modo se determinan las toneladas de $\mathrm{CO}_{2}$ equivalentes, teniendo en cuenta los siete GEI considerados en el GHG Protocol $\left(\mathrm{CO}_{2}, \mathrm{CH}_{4}, \mathrm{~N}_{2} \mathrm{O}\right.$, ozono troposférico $\left(\mathrm{O}_{3}\right)$, CFC-12, HFCC-22 y hexafluoruro de azufre $\mathrm{SF}_{6}$ ) (World Resources Institute y World Business Council for Sustainable Development, 2013).

El interés por determinar las emisiones de GEI en la actualidad se debe no solo a sus múltiples efectos, como la alteración de la composición química del aire, la contaminación térmica (causa del efecto invernadero acentuado) y los riesgos para la salud humana, sino también a la oportunidad que ofrece a las organizaciones en términos de promoción del ahorro financiero y la competitividad (Dangelico y Pujari, 2010). Aunque limitar los efectos del cambio climático significa un trabajo conjunto y es necesario para lograr el desarrollo sostenible y la equidad, hay que tener en cuenta las necesidades individuales, ya que las contribuciones pasadas y futuras de los países a la acumulación de GEI presente en la atmósfera son diferentes, y cada país se enfrenta a desafíos y circunstancias especiales; por tanto, tienen también diferentes capacidades para abordar la mitigación y la adaptación (IPCC, 2014). Por ejemplo, Colombia podría convertirse en uno de los cuarenta países con mayor responsabilidad histórica en la generación de emisiones de GEI por la deforestación, así las contribuciones reportadas en la última comunicación ante el IPCC reflejen que el país hace aportes muy bajos a las emisiones globales. En 2010, Colombia contribuyó con el $0.46 \%$ de las emisiones a escala global, y se estima que el aumento será cercano al $50 \%$ para el año 2030 (García, Barrera, Gómez y Suárez, 2015). Por esto, el país se comprometió con la disminución de emisiones de GEI con una meta de reducción del $30 \%$ para el 2030. Para cumplir este propósito se estableció el desarrollo de inventarios anuales que incluyan los cuatro principales grupos propuestos por el IPCC: energía, procesos industriales, desechos y agricultura, silvicultura y otros usos de la tierra. No obstante, para lograr esta meta se debe contemplar la cooperación de diferentes instituciones, agremiaciones y comunidades, entre otros actores, ya que el diseño de la política climática está influido por la manera en que los individuos y las organizaciones perciben los riesgos y las incertidumbres, y la manera como los toman en cuenta.

En el contexto mencionado, y teniendo en cuenta que está bien documentado que las instituciones de educación superior (IES) a escala mundial son actores influyentes tanto en la formulación de políticas locales y nacionales como en la de información que se hace llegar a la sociedad mediante la investigación y la educación de los graduados sobre las consecuencias del cambio climático (Etzkowitz, 1998), las IES pueden ser incubadoras exitosas de innovación y origen de múltiples iniciativas de sostenibilidad (Robinson, Tewkesbury, Kemp y Williams, 2017). Colombia ha situado la educación como principal prioridad para mejorar el desarrollo económico y social de la nación de manera sostenible. Esto incluye espacios educativos que respondan a problemáticas actuales, como el cambio climático (Mineducación, 2016). Sin embargo, comparadas con las de otros países, en Colombia aún son escasas las IES que reportan inventarios de GEI, razón por la cual existe poca documentación sobre las emisiones de este sector. En Estados Unidos, universidades como la de Pensilvania y Middlebury realizaron su inventario y reportaron las emisiones totales de sus campus. Estas instituciones contemplaron las emisiones asociadas a infraestructura y desarrollo de las diferentes actividades. Los estudios concluyeron que las principales fuentes de contaminación eran la calefacción, el uso de energía eléctrica, el uso de combustibles en los diversos medios de transporte y los residuos sólidos generados en actividades diarias (Isham, del Negro y Bission, 2003; Mondejar, Viñoles, Bastante, Collado y Capuz, 
2011), en tanto que en Europa la Universidad Noruega de Ciencia y Tecnología determinó su huella de carbono $(\mathrm{HC})$, por medio de un modelo ambiental de relación de entradas y salidas, que reportó emisiones de 4.6 t de $\mathrm{CO}_{2}$ e en el período de estudio, y mostró, además, menores emisiones en los departamentos de ciencias sociales y humanidades que en los de ciencias naturales e ingeniería. Estos resultados fueron la base para determinar que la Universidad necesitaba generar diferentes estrategias de mitigación, dependiendo de los comportamientos y actividades realizadas por áreas (Larsen, Pettersen, Solli y Hertwich, 2013).

De esta manera, los estudios de inventario de GEI en instituciones universitarias de diferentes países han logrado identificar los impactos relacionados con el consumo energético y de recursos, como agua, materiales para construcción, papel y combustibles fósiles, o con el manejo inadecuado de los desechos generados, como también el bajo compromiso institucional y de los diversos actores, que probablemente involucren este sector como contribuyente al calentamiento global (Agüero y César, 2014). Por ello, en la actualidad se observa un incremento de los estudios para la determinación del inventario de GEI de las instituciones de educación superior para responder a la necesidad de comprender y transformar las operaciones que ejecutan, además de posicionarse como una actividad responsable y comprometida con el ambiente. En este contexto, este trabajo tuvo como objetivo estimar el inventario de GEI de la Utadeo con el fin de proporcionar información sobre los aportes de la institución al calentamiento global y, adicionalmente, identificar los puntos críticos de emisión.

\section{MATERIALES Y MÉTODOS}

\section{Procedimiento general de análisis}

Se emplearon, como método de evaluación, los procedimientos estandarizados en la Iso 14064-1 (2006). Este protocolo describe los procesos para el inventario de GEI organizacional, y comprende las siguientes etapas: presentación de la institución universitaria, definición de los límites organizacionales (administrativo, financiero, académico y logístico) y operativos e identificación de las fuentes de emisión en la universidad, selección del método de cálculo y elección de factores de emisión, para el posterior análisis y reporte de los resultados.

\section{Presentación de la organización y el alcance}

El estudio se enmarca en las actividades educativas y administrativas de la Utadeo, sede Bogotá, durante el año 2015. Se estimaron las emisiones de GEI, en $\mathrm{CO}_{2}$ e generadas en el período considerado, contemplando los procesos propios de una institución de educación superior, excluyendo transporte de los estudiantes a la universidad, debido a falta de información. La Universidad se encuentra ubicada en la ciudad de Bogotá, D. C., en la localidad de Santa Fe, y cuenta con un campus constituido por biblioteca, museos, estudios de audiovisuales, laboratorios y talleres, auditorios, centros de investigación, salones de clase, salas de cómputo, zonas verdes y espacios deportivos. La sede ofrece veintisiete programas de pregrado, diecisiete especializaciones, dieciocho programas de maestría y dos de doctorado en el conjunto de sus facultades.

La población total en el año 2015 fue de 13238 individuos, entre los que hay 11256 estudiantes y 1982 entre personal docente, administrativo y de servicios.

\section{DEFINICIÓN DE LOS LÍMITES Y FUENTES DE EMISIÓN}

\section{Límites}

En este estudio se eligió el año 2015 como límite temporal, debido a la disponibilidad de datos de inventario relativos a este período. El límite espacial fue determinado por la totalidad del campus universitario, que se encuentra organizado en bloques de aulas de clase, administración y talleres, entre otros (tabla 1)

La Universidad cuenta con $69904.18 \mathrm{~m}^{2}$ de área construida y 33 módulos, constituidos por 1058 espacios (tabla 1). 
Tabla 1. Espacios físicos de la Universidad y área total construida

\begin{tabular}{ccc}
\hline Tipo de espacio & Número de espacios & Área construida $\left(\mathbf{m}^{2}\right)$ \\
\hline Aulas de clase & 194 & 9308.38 \\
Laboratorios & 38 & 2572.28 \\
Salas de tutoría & 5 & 44.31 \\
Auditorios & 4 & 1556.40 \\
Bibliotecas & 1 & 2714.88 \\
Salas de cómputo (37) y audiovisuales (18) & 55 & 2678.03 \\
Oficinas & 501 & 9833.64 \\
Taller & 24 & 1798.09 \\
Almacenamiento & 1 & 2723.71 \\
Servicios asistenciales & 9 & 255.36 \\
Cafeterías & 5 & 1266.85 \\
Servicios sanitarios & 213 & 2159.69 \\
Total & 9 & 32992.56 \\
Otros (circulación interna, estructura y técnicos) & 1058 & 69904.18 \\
\hline
\end{tabular}

Fuente: Oficina de Desarrollo Físico, Utadeo

\section{Límites operacionales}

Los límites operacionales se identificaron a partir de las fuentes de emisión de GEI, y se clasificaron en tres alcances, relacionados con actividades desarrolladas en los límites organizacionales establecidos anteriormente.

Alcance I: se incluyeron los procesos de consumo de combustible de fuentes fijas, como diésel, para el funcionamiento de las plantas eléctricas de la Universidad y el consumo de gas natural en laboratorios y cafeterías.

Alcance II: se consideraron las emisiones relacionadas con el consumo de energía en actividades administrativas y académicas, como iluminación, funcionamiento de equipos de cómputo, televisores, maquinaria, equipos de laboratorio y talleres.

Alcance III: este alcance, aunque es opcional en la metodología propuesta por la ISO 14064-1, fue incluido en el estudio. Se consideraron los siguientes procesos: viajes aéreos realizados por docentes y administrativos, consumo de agua en el funcionamiento de los baños, actividades de riego, limpieza, lavado de fachadas y zonas comunes, consumo de papel en actividades académicas y administrativas, generación de residuos peligrosos y reciclables e infraestructura construida.

\section{Identificación de las fuentes de emisión}

Las fuentes de emisión fueron establecidas a partir de las principales actividades que se realizan dentro de la Utadeo. Se inventarió el consumo de diésel, consumo de gas natural, de energía eléctrica, de agua y de papel, transporte aéreo, generación de residuos reciclables y peligrosos e infraestructura construida.

\section{SELECCIÓN DEL MÉTODO DE CÁLCULO Y RECOLECCIÓN DE DATOS}

Para determinar el inventario de GEI se elaboró una hoja de cálculo en Excel, donde se anotarían los datos de inventario siguiendo el estándar ISO 14064-1.

El inventario de las diferentes actividades académicas y administrativas se adquirió mediante el acceso a diferentes fuentes de información reportadas por la Universidad y centralizadas en la Dirección de Desarrollo Organizacional.

\section{Factores de emisión}

Los factores de emisión utilizados para cada una de las actividades y sus respectivas fuentes se muestran en la tabla 2. 
Tabla 2. Factores de emisión para el cálculo del inventario de GEI

\begin{tabular}{|c|c|c|c|c|}
\hline \multicolumn{3}{|c|}{ Actividad } & Factor de emisión de $\mathrm{CO}_{2} \mathrm{e}$ & $\begin{array}{c}\text { Fuente de factor de } \\
\text { emisión }\end{array}$ \\
\hline \multicolumn{3}{|l|}{ Consumo de diésel } & 10.2765 kg CO $2 \mathrm{e} . / \mathrm{gal}$ & \multirow{3}{*}{$\begin{array}{c}\text { Unidad de } \\
\text { Planeación Minero } \\
\text { Energética }^{1}\end{array}$} \\
\hline \multicolumn{3}{|c|}{ Consumo de gas natural } & $1.9801 \mathrm{~kg} \mathrm{CO} 2 \mathrm{e} . / \mathrm{m}^{3}$ & \\
\hline \multicolumn{3}{|c|}{ Consumo de energía eléctrica } & $0.221 \mathrm{~kg} \mathrm{CO} 2 \mathrm{e} . / \mathrm{kWh}$ & \\
\hline \multirow{3}{*}{ Transporte aéreo } & \multicolumn{2}{|l|}{ Vuelos nacionales } & $0.205 \mathrm{~kg} \mathrm{CO} \mathrm{e}_{2} / \mathrm{km}$ recorrido & \multirow{3}{*}{ PUCC $^{2} ;$ DEFRA $^{3}$} \\
\hline & \multirow{2}{*}{ Vuelos internacionales } & Cortos* & $0.116 \mathrm{~kg} \mathrm{Co} \mathrm{e}_{2} \mathrm{e} / \mathrm{km}$ recorrido & \\
\hline & & Largos** & $0.135 \mathrm{~kg} \mathrm{Co} \mathrm{e}_{2} \mathrm{e} / \mathrm{km}$ recorrido & \\
\hline \multicolumn{3}{|l|}{ Consumo de agua } & $0.1427 \mathrm{~kg} \mathrm{CO}{ }_{2} \mathrm{e} . / \mathrm{m}^{3}$ & $\mathrm{uco}^{4}$ \\
\hline \multicolumn{3}{|l|}{ Consumo de papel } & $1.30 \mathrm{~kg} \mathrm{CO} 2 \mathrm{e} . / \mathrm{kg}$ & $\mathrm{EPA}^{5}$ \\
\hline \multirow{8}{*}{$\begin{array}{l}\text { Generación de } \\
\text { residuos reciclados }\end{array}$} & \multicolumn{2}{|l|}{$\mathrm{PET}^{* * *}$} & $2.538 \mathrm{~kg} \mathrm{CO} 2 \mathrm{e} . / \mathrm{kg}$ residuo & \multirow{5}{*}{ ASIPLA $^{6}$} \\
\hline & \multicolumn{2}{|l|}{ Hierro } & $2.9 \mathrm{~kg} \mathrm{CO} 2 \mathrm{e} . / \mathrm{kg}$ residuo & \\
\hline & \multicolumn{2}{|l|}{ Polipropileno } & $1.343 \mathrm{~kg} \mathrm{CO} 2 \mathrm{e} . / \mathrm{kg}$ residuo & \\
\hline & \multicolumn{2}{|l|}{ Vidrio } & 0.04 kg co 2 e. $/ \mathrm{kg}$ residuo & \\
\hline & \multicolumn{2}{|l|}{ Aluminio } & $9.13 \mathrm{~kg} \mathrm{CO} 2 \mathrm{e} . / \mathrm{kg}$ residuo & \\
\hline & \multicolumn{2}{|l|}{ Papel + cartón } & $0.55 \mathrm{~kg} \mathrm{CO} 2 \mathrm{e} . / \mathrm{kg}$ residuo & \multirow{2}{*}{ DEFRA } \\
\hline & \multicolumn{2}{|l|}{ Plástico } & $0.035 \mathrm{~kg} \mathrm{CO} 2 \mathrm{e} . / \mathrm{kg}$ residuo & \\
\hline & \multicolumn{2}{|l|}{ Vasos de cartón } & $10.15 \mathrm{~kg} \mathrm{CO} 2 \mathrm{e} . / \mathrm{kg}$ residuo & $\mathrm{ANIQ}^{7}$ \\
\hline \multirow{9}{*}{$\begin{array}{l}\text { Generación de } \\
\text { residuos peligrosos }\end{array}$} & \multicolumn{2}{|l|}{$\mathrm{RAEE} * * * *$} & $0.0846 \mathrm{~kg} \mathrm{CO} \mathrm{C}_{2} \mathrm{e} / \mathrm{kg}$ residuo & \multirow{6}{*}{ uco } \\
\hline & \multicolumn{2}{|c|}{ Biosanitarios y residuos sin categorizar } & $0.0502 \mathrm{~kg} \mathrm{co} 2 \mathrm{e} . / \mathrm{kg}$ residuo & \\
\hline & \multicolumn{2}{|c|}{ Disolventes } & $0.0991 \mathrm{~kg} \mathrm{Co} 2 \mathrm{e} . / \mathrm{kg}$ residuo & \\
\hline & \multicolumn{2}{|c|}{ Materiales y envases contaminados } & $0.0262 \mathrm{~kg} \mathrm{CO} \mathrm{C}_{2} \mathrm{e} / \mathrm{kg}$ residuo & \\
\hline & \multicolumn{2}{|c|}{ Ácidos, bases y soluciones } & $0.0677 \mathrm{~kg} \mathrm{CO} \mathrm{e}_{2} \mathrm{e} / \mathrm{kg}$ residuo & \\
\hline & \multicolumn{2}{|l|}{ Aceites } & $0.0347 \mathrm{~kg} \mathrm{CO} \mathrm{e}_{2} / \mathrm{kg}$ residuo & \\
\hline & \multicolumn{2}{|l|}{ Pesticidas } & $7.37 \mathrm{~kg} \mathrm{CO} \mathrm{e}_{2} \mathrm{e} / \mathrm{kg}$ residuo & Carbon Trust \\
\hline & \multicolumn{2}{|l|}{ Residuos orgánicos } & $0.365 \mathrm{~kg} \mathrm{CO}{ }_{2} \mathrm{e} . / \mathrm{kg}$ residuo & DEFRA \\
\hline & \multicolumn{2}{|l|}{ Tóner } & $2.76 \mathrm{~kg} \mathrm{CO} 2 \mathrm{e} . / \mathrm{kg}$ residuo & ASIPLA \\
\hline Aguas residuales inc & ustriales & & $5,63 \mathrm{~kg} \mathrm{CO}{ }_{2} \mathrm{e} . / \mathrm{DQO}$ & IPCC \\
\hline Infraestructura cons & truida & & $520 \mathrm{~kg} \mathrm{CO} / \mathrm{m}^{2}$ construido & \\
\hline
\end{tabular}

${ }^{1}$ Unidad de Planeación Minero Energética; ${ }^{2}$ Pontifica Universidad Católica de Chile; ${ }^{3}$ Department for Environment, Food \& Rural Affairs; ${ }^{4}$ Universidad de Córdoba; ${ }^{5}$ Environmental Protection Agency; ${ }^{6}$ Análisis del impacto de los gases de efecto invernadero en el ciclo de vida de los embalajes y otros productos plásticos en Chile V1.0; ${ }^{7}$ Asociación Nacional de la Industria Química.

* Distancias menores a $2000 \mathrm{~km}$

** Distancias mayores a $2000 \mathrm{~km}$

$* * *$ Tereftalato de polietileno

$* * * *$ Residuos de aparatos electrónicos y eléctricos

PCGN 2 O: 298

PCG CFC-11: 4750

PCG CFC-12: 10900

PCG Halon-1301: 7140

PCG Carbon Tetrachloride: 1400 
El factor de emisión empleado para vasos de cartón fue establecido por medio del estudio mexicano "Análisis de ciclo de vida de vasos desechables en México", en el que se determinó el aporte a la categoría de calentamiento global para 1600 millones de vasos de papel plastificado de $10 \mathrm{oz}$. La carga ambiental potencial en el ciclo de vida fue de $95809900 \mathrm{~kg}$ de $\mathrm{CO}_{2} \mathrm{e}$ por la unidad funcional. Sin embargo, para el uso de un factor de emisión en este estudio fue necesario pasarlo a kg de vasos, por lo cual se determinó un peso promedio de vasos de 10 onzas ( $0.0059 \mathrm{~kg} /$ vaso), y luego de determinar la contribución en $\mathrm{CO}_{2}$ e de la unidad, fue llevado a kilogramo de vasos, como se muestra en la ecuación 1.

\section{Factor de emisión $\left(\mathrm{kg} \mathrm{CO}_{2} \text { Eq. }\right)_{1 \text { pieza }}=\frac{95809900 \mathrm{~kg} \mathrm{CO} 2 \mathrm{Eq} .}{1600000000} \quad E_{\text {C. }}{ }^{l}$ $=0.0599 \frac{\mathrm{kgCO}_{2} \mathrm{Eq} .}{\text { pieza }} * 169.5$ vasos $(1 \mathrm{~kg}$ de vasos $)=10.15 \mathrm{~kg} \mathrm{co} 2 \mathrm{e} . / \mathrm{kg}$}

Para el caso de aguas residuales industriales, el factor de emisión se determinó a partir de las directrices establecidas por el IPCC para aguas residuales, empleando la ecuación 2.

$$
\mathrm{FE}_{\mathrm{ARI}}=\mathrm{CMP}_{\mathrm{CH}_{4}} * \mathrm{FC}_{\mathrm{CH}_{4}} * \mathrm{PCG}_{\mathrm{CH}_{4}}
$$

Donde:

$\mathrm{FE}_{\mathrm{ARI}}$ : factor de emisión para tratamiento de las aguas residuales industriales

$\mathrm{CMP}_{\mathrm{CH}_{4}}$ : capacidad máxima de producción de metano en las aguas residuales industriales (el valor por defecto es $0.25 \mathrm{~kg}$ de $\mathrm{CH} 4{ }^{*} \mathrm{~kg}$ DBO5)

$\mathrm{FC}_{\mathrm{CH}_{4}}$ : factor de corrección para el metano (el valor por defecto es 0.9)

$\mathbf{P C G}_{\mathrm{CH}_{4}}$ : potencial de calentamiento global del metano (25)

\section{RESULTADOS Y DISCUSIÓN}

La tabla 3 muestra los resultados del inventario de GEI de la Utadeo en cada uno de los alcances, con un total de $1688.365 \mathrm{t} \mathrm{co}_{2} \mathrm{e}$ y correspondientes a $0.15 \mathrm{t}$ $\mathrm{CO}_{2}$ e por estudiante para el año 2015. Del total de emisiones, $8.18 \%$ proviene del alcance I, $37.25 \%$ del II y $54.57 \%$ del III. Estos resultados son inferiores a los encontrados en estudios de diferentes instituciones. En la Universidad de Montfort, en Inglaterra, fueron determinadas $2.3 \mathrm{t} \mathrm{CO}_{2}$ e por estudiante (Ozawa-Meida, Brockway, Letten, Davies y Fleming, 2013); en la Universidad Noruega de Ciencia y Tecnología, 4.6 t $\mathrm{CO}_{2}$ e por estudiante (Larsen et al., 2013), y en la Universidad Cas-
tilla-La Mancha, en España, 0.74 t $\mathrm{CO}_{2}$ e por estudiante (Gómez, Cadarso y Monsalve, 2016). Sin embargo, es necesario tener en cuenta que los dos primeros estudios contemplaron el transporte de los estudiantes a las universidades, mientras que en el presente estudio y en el de la Universidad Castilla-La Mancha este parámetro no fue involucrado. Las cargas ambientales por uso de transporte pueden incrementar ampliamente el inventario de GEI de la Utadeo, ya que los estudiantes se desplazan, en su mayoría, en servicio público desde diferentes barrios de Bogotá o localidades de la sabana, y su servicio en general es poco eficiente, aspecto relacionado con la antigüedad y uso de gasolina y diésel como combustible. Según reporta la Secretaría de Movilidad de Bogotá D. C. (2015), el 76.7 \% de los vehículos de servicio público tipo bus corresponden a modelos 2003 y posteriores, cuyo combustible es diésel. En cuanto a busetas, el $80 \%$ funcionan con diésel y el $20 \%$, que son modelos antiguos (1992 y anteriores), operan con gasolina, y los microbuses son modelos fabricados entre 1993 y 2013, y usan diésel y gasolina como principal fuente de combustible.

En el alcance III, el transporte aéreo juega un papel fundamental en los aportes al inventario, con un segundo lugar en aportes después de infraestructura. Las emisiones de GEI por este aspecto están vinculadas con los viajes nacionales e internacionales de pro- 
fesores, estudiantes y administrativos. Los destinos nacionales visitados con mayor frecuencia en el año 2015 fueron las ciudades de Santa Marta y Cartagena, debido a que existen dos sedes de la universidad en estas localidades. También se reportaron viajes frecuentes a Cúcuta y Neiva, asociados al desarrollo de convenios con instituciones de educación en estas regiones. En cuanto a viajes internacionales, los destinos a los que se viajó con mayor frecuencia fueron París, Monterrey y Santiago de Chile, debido a compromisos institucionales como participación en congresos o búsqueda de convenios, entre otros. Las emisiones de GEI por transporte aéreo de personal y estudiantes de la Utadeo se calcularon en 136.641 t $\mathrm{CO}_{2} \mathrm{e}$, lo que se traduce en una contribución del $14.83 \%$ en este alcance. Los vuelos internacionales aportan mayor cantidad de toneladas de $\mathrm{CO}_{2}$ e que los nacionales, debido a que viajes realizados al exterior implican mayor gasto de combustible (queroseno), ya que las cargas ambientales de la aviación asociadas al forzamiento radiativo (FR) se encuentran relacionadas con las emisiones de $\mathrm{CO}_{2} \mathrm{y} \mathrm{NO}_{x^{\prime}}$ principalmente (Lee, Fahey, Forster, Newton, Wit, Lim, Owen y Sausen, 2009). Los resultados aquí encontrados son similares a los cálculos del inventario de GEI del Instituto de Ingeniería de la Universidad Autónoma de México (Güereca, Torres y Noyola, 2013), la Universidad de Córdoba (Toro, Gomera, Aguilar, Guijarro, Antúnez y Vaquero, 2015) y la Universidad Politécnica Salesiana, en Ecuador (Dávila y Varela, 2014). En ellos también se determinó que la mayor carga estaba asociada a los viajes en avión, entre los diferentes medios de transporte contemplados, lo cual demuestra que en el alcance III, la movilidad es una de las actividades que más aportes hacen a las emisiones de GEI. Sin embargo, se debe considerar que, en este estudio, no se tuvo en cuenta la movilización terrestre de estudiantes, personal administrativo y docente, lo cual le resta la contribución de este alcance al inventario de GEI final. Las emisiones generadas por transporte aéreo son muy cercanas al total generado en el alcance I, con diferencia de $1.52 \mathrm{t} \mathrm{CO}_{2} \mathrm{e}$, que evidencia que los viajes superan el uso de combustible empleado en actividades dentro de la Utadeo.

La Utadeo tiene alto consumo de papel, dadas las diferentes actividades que lo requieren; principalmente, impresión y fotocopiado de documentos en las diver- sas dependencias administrativas y académicas. El consumo total en el año de estudio fue de 19.99 t/año, lo cual representa una contribución de 2,82 \% en el alcance y $25.98 \mathrm{t} \mathrm{CO}_{2}$ e de emisiones. Esto representa un consumo medio de $1.60 \mathrm{~kg} / \mathrm{año}$ de papel por estudiante, valor inferior al determinado en el Instituto de Ingeniería de la Universidad Autónoma de México, que fue de $9 \mathrm{~kg}$ por estudiante, aunque con mínimos aportes al inventario de GEI. Este fue menor a los aportes encontrados para este aspecto en la Universidad de Montfort, en donde calcularon 216 t $\mathrm{CO}_{2}$ e en el periodo académico comprendido entre el 2008 y 2009 (Ozawa-Meida et al., 2013). En cuanto a generación de residuos, la Uta-

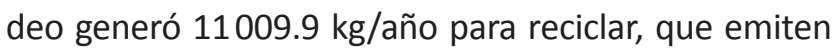
$22.08 \mathrm{t} \mathrm{CO}_{2} \mathrm{e}$, que representa el 2,40 \% (figura 1). Los vasos de cartón fueron el residuo con mayores aportes (8.82 t $\mathrm{CO}_{2}$ e y $0.96 \%$ de contribución). El uso de vasos es alto como resultado de la generalización de su uso en oficinas, venta de café en dispensadores de bebidas y cafeterías en este tipo de envase. Es preciso resaltar que las emisiones por el uso de este tipo de vasos son relevantes, porque el ciclo de vida del producto se caracteriza por llevar a cabo procesos que requieren alto consumo de energía y materias primas en su elaboración (Asociación Nacional de la Industria Química, 2013). Los demás residuos generados resultan de la realización de diversas actividades, como almacenamiento de productos de laboratorios, consumo de bebidas, material de talleres de diseño. La mayoría de los residuos peligrosos proviene de laboratorios de biología y diferentes carreras de ingeniería, talleres de fotografía y mantenimiento. Aunque los aportes de los residuos para reciclaje y peligrosos hacen un aporte mínimo, que corresponde al $3.47 \%$, lo cual concuerda con el estudio realizado por Toro et al. (2015) en la Universidad de Córdoba (España), no se puede subestimar esta carga y, por tanto, se deben buscar programas para hacer más eficiente el uso de insumos y minimizar la extracción de materias primas.

Finalmente, las mayores emisiones en el alcance III estuvieron asociadas a la infraestructura para el desarrollo de las actividades académicas, administrativas y culturales. El total de emisiones fue de 921.382 t $\mathrm{CO}_{2}$ e, con aportes de 54.57 \% (figura 1) al inventario de GEI total. Este resultado es relevante, ya que es acorde con lo expresado por Huang, Weber y 
Matthews (2009) y Downie y Stubbs (2013), quienes expresan que las emisiones ligadas a este alcance pueden representar hasta el $75 \%$ de las emisiones directas e indirectas para la gran mayoría de las empresas. Asimismo, diversos estudios del inventario de GEI de instituciones universitarias coinciden en emisiones más altas desde este alcance. Por ejemplo, Ozawa-Meida et al. (2013) reportan el 79 \% en el inventario de GEI desde este alcance en la Universidad de Montfort, 53 \% en la Universidad de Lancaster y $61 \%$ en la Universidad de York (en el Reino Unido); estos aportes fueron el resultado de consumo de papel en actividades académicas y administrativas, y otras de menor carga, la generación de residuos reciclables y peligrosos. El área construida de la Utadeo corresponde a $69904.18 \mathrm{~m}^{2}$, a la cual se le asignó una vida útil de cincuenta años, que representa $727.003 \mathrm{t} \mathrm{CO}_{2} \mathrm{e}$ (tabla 3), con aportes del $78.90 \%$ (figura 1), en este alcance. Las contribuciones al inventario de GEI por este aspecto se encuentran relacionadas con lo especificado por Mercader, Ramírez de Arellano y Olivares (2012), quienes determinan que el requerimiento de altas cantidades de energía en procesos de explotación y transformación de materiales y la disposición de residuos y emanaciones a la atmósfera, durante el ciclo de vida de las obras y productos, generan altas emisiones. Aunque no se realizó inventario detallado de los materiales y energía utilizados en el proceso de construcción, la determinación de las áreas y la asignación de un período de vida útil permitieron agregar esta entrada al cálculo del inventario de GEI. Con respecto a este parámetro, la Escuela Universitaria Politécnica de Manresa, por medio del modelo de investigación de edificación sostenible (MIES) concuerda con que la estimación de las emisiones asociadas a la vida útil de los edificios representa alta carga ambiental (Jorge y Busquets, 2003).

En segundo lugar, se determinaron los aportes por consumo de energía eléctrica (alcance II) comprados con los de la red de interconexión eléctrica de la ciudad y con el $100 \%$ del aporte. En este alcance se contemplaron las actividades administrativas y académicas que requieren uso de equipos conectados a la red, así como sistemas de iluminación. Las emisiones totales fueron calculadas en $628.833 \mathrm{t} \mathrm{co}_{2} \mathrm{e}$, con un consumo total de $3159.967 \mathrm{kWh}$. El aporte por estas actividades a las emisiones totales (37.25\%) fue representativo, dado que la mayoría de las áreas requieren iluminación para el desarrollo de actividades diurnas o nocturnas. En general, el diseño de aulas y oficinas no permite el uso de luz natural. Además, el funcionamiento constante de equipos que apoyan los aspectos administrativos y académicos diarios tiene alta demanda energética. Este resultado concuerda con lo reportado por Güereca et al. (2013) y Aroonsrimorakot, Yuwaree, Arunlertaree, Hutajareorn y Buadit (2013) para la Universidad Autónoma de México y la Universidad Mahidol de Tailandia, en donde el consumo de energía se sitúa como una de las actividades con mayor carga a las emisiones de $\mathrm{CO}_{2}$ e en instituciones de educación superior, aunque no el principal. Contrario a lo encontrado por Utaraskul (2015) en la Universidad de Suan Sunandha Rajabhat (Tailandia), quien afirma que el alcance II presentó la mayor carga ambiental en el inventario de GEI, con $44 \%$ de contribución, debido principalmente al uso de aparatos electrónicos.

Según el Sistema de Información Eléctrico Colombiano, el país cuenta con una mezcla energética basada en hidroelectricidad como principal fuente de generación de energía y un pequeño aporte de generación térmica. Por esta razón, el factor de emisión es relativamente bajo. La hidroenergía es una de las alternativas que menor impacto tienen en la emisión de GEI. Sin embargo, en el año 2015 hubo un déficit de aportes hídricos, como consecuencia del fenómeno de El Niño, que ocasionó incrementos en la generación de energía térmica en $12.1 \%$ e hidráulica en 0.7 , en comparación con el 2014 (Isagén, 2016). Esto resultó en un factor de emisión superior al del año anterior, posiblemente como consecuencia de la quema de combustibles, entre ellos gas natural, carbón y diésel para el funcionamiento de las centrales térmicas, a lo que se suman aumentos en la demanda de energía. Con base en lo anterior, en la Utadeo, el gasto de electricidad posiblemente se relacione con los episodios de déficit del recurso hídrico ocurridos en 2015 y a la mayor demanda, por el crecimiento de la institución en cuanto a infraestructura y población. Sin embargo, es necesario realizar comparaciones con años anteriores para poder establecer diferencias y desarrollar estrategias de uso eficiente. Además, es posible que el consumo esté relacionado con el uso poco responsa- 
Tabla 3. Inventario de GEI de la UJTL

\begin{tabular}{|c|c|c|c|c|c|c|c|c|c|}
\hline Alcance & \multicolumn{3}{|c|}{ Fuente de emisión } & Cantidad & Unidad & \multicolumn{2}{|c|}{ Factor de emisión } & $\mathrm{T}$ de $\mathrm{CO}_{2} \mathrm{e}$ & Contribución (\%) \\
\hline \multirow{3}{*}{ I } & \multicolumn{3}{|c|}{ Consumo de diésel } & 13070 & gal & \multicolumn{2}{|c|}{10.2765 kg co, e/gal } & 134.314 & 97.2 \\
\hline & \multicolumn{3}{|c|}{$\begin{array}{l}\text { Consumo de gas natural } \\
\text { genérico }\end{array}$} & 1944 & $\mathrm{~m}^{3}$ & \multicolumn{2}{|c|}{$1.9801 \mathrm{~kg} \mathrm{CO} \mathrm{e}_{2} \mathrm{e} / \mathrm{m}^{3}$} & 3.849 & 2.78 \\
\hline & \multicolumn{7}{|c|}{ Total acance I } & 138.163 & 8.18 \\
\hline \multirow[t]{2}{*}{ II } & \multicolumn{3}{|c|}{$\begin{array}{l}\text { Consumo de energía } \\
\text { eléctrica }\end{array}$} & 3159967 & kWh & \multicolumn{2}{|c|}{$0.221 \mathrm{~kg} \mathrm{CO}{ }_{2} \mathrm{e} / \mathrm{kWh}$} & 628.833 & 100 \\
\hline & \multicolumn{7}{|c|}{ Total alcance II } & 628.833 & 37.25 \\
\hline \multirow{9}{*}{ III } & \multirow{3}{*}{$\begin{array}{l}\text { Transporte } \\
\text { aéreo }\end{array}$} & \multicolumn{2}{|l|}{$\mathrm{VN}^{*}$} & 257332.51 & \multirow{3}{*}{$\mathrm{km}$} & \multicolumn{2}{|c|}{$\begin{array}{l}0.205 \mathrm{~kg} \mathrm{CO}{ }_{2} \mathrm{e} / \mathrm{km} \\
\text { recorrido }\end{array}$} & & \\
\hline & & \multirow{2}{*}{$\mathrm{VI} * *$} & C & 25843.59 & & \multicolumn{2}{|c|}{$\begin{array}{l}0.116 \mathrm{~kg} \mathrm{CO} \mathrm{e}_{2} / \mathrm{km} \\
\text { recorrido }\end{array}$} & 136.641 & 14.82 \\
\hline & & & $\mathrm{L}$ & 599188.48 & & \multicolumn{2}{|c|}{$\begin{array}{l}0.135 \mathrm{~kg} \mathrm{CO} \mathrm{e}_{2} \mathrm{e} / \mathrm{km} \\
\text { recorrido }\end{array}$} & & \\
\hline & \multicolumn{3}{|c|}{ Consumo de agua } & 29020 & $\mathrm{~m}^{3}$ & \multicolumn{2}{|c|}{$0.1427 \mathrm{~kg} \mathrm{CO} \mathrm{e}_{2} / \mathrm{m}^{3}$} & 4.141 & 0.45 \\
\hline & \multicolumn{3}{|c|}{ Consumo de papel } & 19986 & $\mathrm{~kg}$ & \multicolumn{2}{|c|}{$1.30 \mathrm{~kg} \mathrm{CO}, \mathrm{e} / \mathrm{kg}$} & 25.982 & 2.82 \\
\hline & \multicolumn{3}{|c|}{ PET } & 635.6 & & 2.538 & & 1.613 & 0.175 \\
\hline & \multicolumn{3}{|l|}{ Hierro } & 345 & & 2.9 & & 1.001 & 0.11 \\
\hline & \multicolumn{3}{|c|}{ Polipropileno } & 141.4 & & 1.343 & & 0.190 & 0.02 \\
\hline & Vidrio & & & 328 & & 0.04 & $\mathrm{~kg} \mathrm{CO} 2 \mathrm{e} / \mathrm{kg}$ & 0.013 & 0.0014 \\
\hline \multirow{6}{*}{$\begin{array}{l}\text { Residuos } \\
\text { para } \\
\text { reciclaje }\end{array}$} & Papel y cart & & & 7318 & Kg & 0.55 & residuo & 4.025 & 0.44 \\
\hline & Plástico & & & 672 & & 0.035 & & 0.024 & 0.0025 \\
\hline & Vasos de ca & rtón & & 868.9 & & 10.15 & & 8.819 & 0.96 \\
\hline & Aluminio & & & 701 & & 9.13 & & 6.400 & 0.70 \\
\hline & RAEE & & & 1151 & & 0.0846 & & 0.097 & 0.01 \\
\hline & $\begin{array}{l}\text { Biosanitaric } \\
\text { categorizar }\end{array}$ & s y $\sin$ & & 2569 & & 0.0502 & & 0.129 & 0.014 \\
\hline Residuos & Disolventes & & & 262.9 & & 0.0991 & & 0.026 & 0.0082 \\
\hline peligrosos & $\begin{array}{l}\text { Materiales } \\
\text { contaminac }\end{array}$ & $\begin{array}{l}\text { y envas } \\
\text { los }\end{array}$ & & 206.1 & & 0.0262 & $\mathrm{~kg} \mathrm{CO}{ }_{2} \mathrm{e} / \mathrm{kg}$ & 0.005 & 0.00059 \\
\hline & $\begin{array}{l}\text { Ácidos, bas } \\
\text { soluciones }\end{array}$ & es y & & 3184.6 & kg & 0.0677 & residuo & 0.216 & 0.023 \\
\hline & Aceites & & & 1 & & 0.0347 & & 0.0000347 & 0.0038 \\
\hline & Pesticida in & específi & & 312.8 & & 7.37 & & 2.305 & 0.25 \\
\hline & Residuos or & gánicos & & 4 & & 0.365 & & 0.001 & 0.00016 \\
\hline & Tóner & & & 925 & & 2.76 & & 2.553 & 0.28 \\
\hline & $\begin{array}{l}\text { Aguas resid } \\
\text { industriales }\end{array}$ & uales & & 32.6 & $\mathrm{~kg}$ & 5.63 & $\begin{array}{l}\mathrm{kg} \mathrm{CO} 2 \mathrm{e} / \\
\text { DQO }\end{array}$ & 0.183 & 0.02 \\
\hline & $\begin{array}{l}\text { Infraestruc } \\
\text { construida }\end{array}$ & & & 69904.18 & $m^{2}$ & 520 & $\begin{array}{l}\mathrm{kg} \mathrm{CO}_{2} \mathrm{e} / \mathrm{m}^{2} \\
\text { construido }\end{array}$ & 727.003 & 79 \\
\hline & Total alcanc & e III & & & & & & 921.369 & 54.57 \\
\hline & Inventario & e GEI & & & & & & 1688.365 & 100 \\
\hline
\end{tabular}

* Viajes nacionales

* Viajes internacionales 
ble: a menudo se dejan encendidas las luces de aulas, baños y diferentes espacios, así como equipos electrónicos sin apagar. Rodas (2014) reporta que las instituciones de educación superior no son directamente responsables de las emisiones generadas por el consumo de energía eléctrica, ya que las emisiones provienen de procesos de producción y distribución de los generadores del servicio, así que ellos se pueden considerar los responsables directos de las emisiones. No obstante, al ser proveedores de servicios, la generación de emisiones se traslada a las instituciones.

Por último, en el alcance I, el consumo de diésel (13070 galones) estuvo asociado al funcionamiento de las plantas eléctricas durante los cortes de energía, en tanto que el consumo de gas natural $\left(1944 \mathrm{~m}^{3}\right)$ se atribuyó al funcionamiento de las cafeterías y los laboratorios. Se contempló únicamente el consumo de combustibles para estos procesos, dado que en la Utadeo no se registra el consumo energético en equipos de climatización ni vehículos. No obstante, diversos estudios internacionales demuestran que el consumo de combustibles en calefacción, aire acondicionado y movilidad de vehículos propios son fuentes de emisión representativas en este alcance (Isham et al., 2003). El total de emisiones fue de $138.163 \mathrm{t} \mathrm{CO}_{2} \mathrm{e}$, con aportes del $97.21 \%$ por uso de diésel y $2.78 \%$ por consumo de gas natural genérico (tabla 3 ). En efecto, las mayores emisiones estuvieron asociadas al uso del combustible para seis plantas de generación de electricidad, necesarias como medida de contingencia para los períodos de interrupción del fluido en la red eléctrica. El consumo de diésel representa una alta carga ambiental, ya que los procesos de combustión incrementan el contenido de residuos minerales y emisiones de humo. Por esto, se considera el gas natural la mejor alternativa respecto a otro tipo de combustibles, incluido el diésel. La combustión del gas produce una mínima cantidad de residuos y funciona como fuente de energía directa en actividades productivas, es decir, no requiere de transformaciones, como los derivados del petróleo (Minetad, 2016); por esta razón tiene un factor de menor emisión. Estos varían no solamente de acuerdo con el tipo de combustible, sino con la actividad en la que se aplique su proceso de combustión y la tecnología utilizada para tal fin (Academia Colombiana de Ciencias Exactas, Físicas y Naturales, 2013).

Figura 1. Contribución de los alcances I, II y III a las emisiones de $\mathrm{CO}_{2}$ en el inventario de GEI

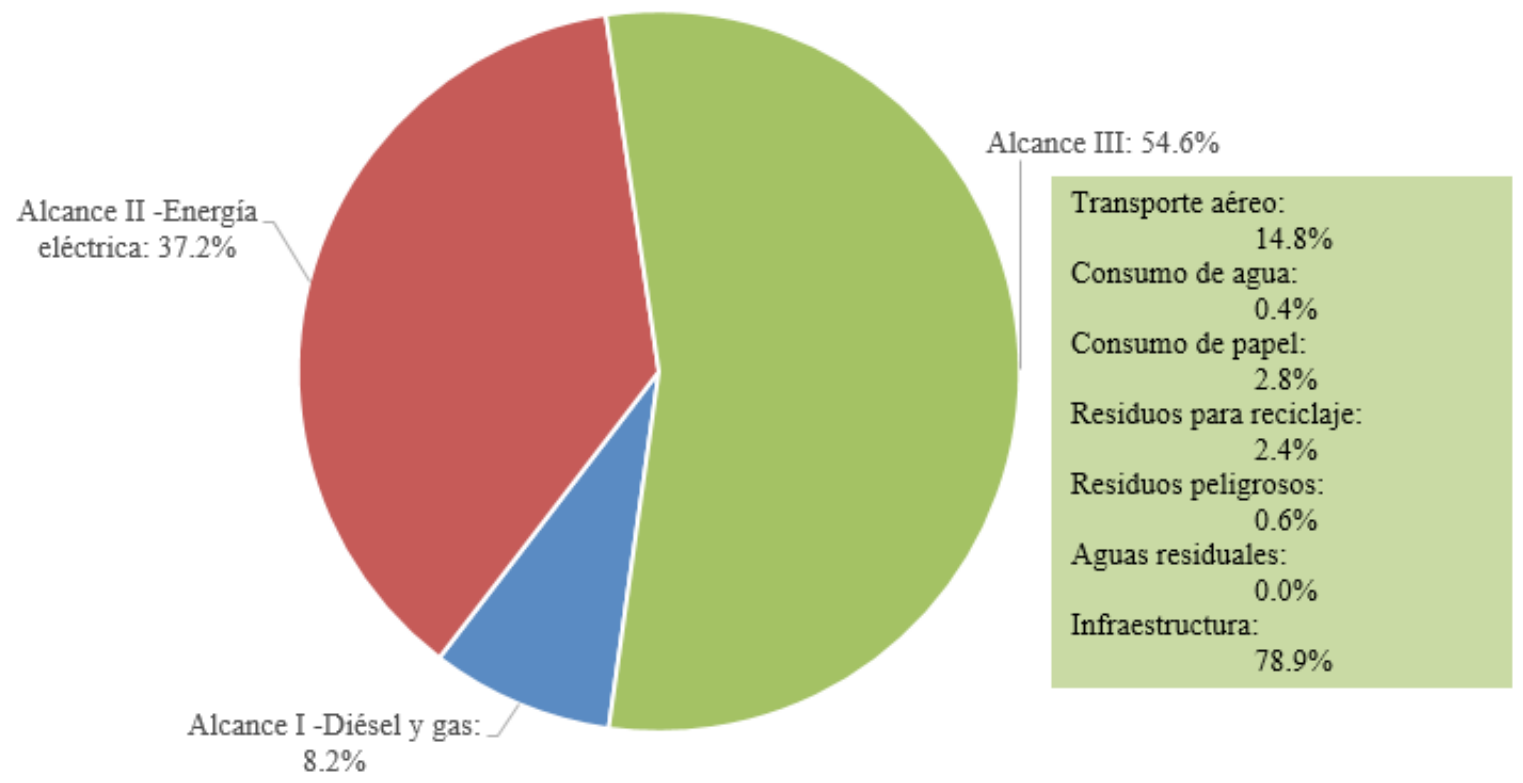


El consumo de gas natural no fue significativo, dado que la Utadeo, en el 2015, contaba con cinco cafeterías, de las cuales solo dos hacían uso del recurso para la preparación de alimentos. En laboratorios el gas solo se utilizó para los mecheros, que no requieren gran cantidad de combustible para su funcionamiento. De esta manera, este alcance presentó aportes del 8.183 \% (figura 1) al inventario de GEI, con la menor contribución a las emisiones de $\mathrm{CO}_{2} \mathrm{e}$.

En resumen, el inventario de GEI de la Utadeo recibe la mayor contribución por el alcance III, seguido por el II y, por último, el alcance I. Esto indica que la Utadeo se caracterizó por tener entre sus actividades educativas procesos relacionados con los tres alcances, lo que evidencia el consumo de diversos recursos y la generación de residuos. Es importante resaltar la necesidad de construir una base de datos actualizada que contemple la totalidad de datos requeridos para la determinación de un inventario de GEI más ajustado a la realidad.

\section{CONCLUSIONES}

El inventario de GEI de la Utadeo, sede Bogotá, en el año 2015 fue de 1688.365 t de $\mathrm{CO}_{2} \mathrm{e}$, que corresponde a la sumatoria de los alcances I, II y III.

Las emisiones directas de GEI están asociadas al consumo de combustibles fósiles, y las indirectas al consumo de energía eléctrica, viajes aéreos, consumo de agua y papel, generación de residuos peligrosos, y residuos para reciclaje, aguas residuales industriales y construcción.

En el inventario de GEI, el alcance que más toneladas de co2e generó fue el III, principalmente por actividades de construcción y transporte aéreo.

Entre las actividades realizadas en instituciones de educación superior, aquellas que involucran el consumo de combustibles fósiles son las de mayor aporte a la emisión de GEI.

\section{RECOMENDACIONES}

Es importante que la Utadeo haga anualmente inventario de sU GEI, para poder realizar comparaciones respecto al año base y saber si se ha reducido, mantenido o aumentado la cantidad de emisiones.

Se recomienda llevar registros de cada uno de los consumos que se dan en la institución debido a sus diferentes actividades educativas y de la generación de residuos, con el fin de integrar en el cálculo de la huella todas las actividades y obtener información más confiable y real. Además, como estrategia para disminuir la cantidad de emisiones, conviene realizar campañas de sensibilización, entre toda la comunidad universitaria, acerca del uso de los recursos y la generación de residuos.

\section{REFERENCIAS}

Academia Colombiana de Ciencias Exactas, Físicas y Naturales (2013). Factor de emisión de los combustibles colombianos: informe final. Disponible en http://www.siame.gov.co/siame/ documentos/documentacion/mdl/HTML/18_ FECOC.htm.

Agüero, C. y César, E. (2014). Elaboración de un protocolo de gestión ambiental para micro, pequeñas y medianas empresas de turismo rural comunitario en Costa Rica, que facilite el proceso de reducción de huella de carbono en sus actividades. Revista de Investigación en Turismo y Desarrollo Local, 7(16), 55-59.

Aroonsrimorakot, S., Yuwaree, C., Arunlertaree, C., Hutajareorn, R. y Buadit, T. (2013). Carbon footprint of faculty of environment and resource studies. APCBEE Procedia, 5, 175-180.

Asociación Nacional de la Industria Química (2013). Análisis de ciclo de vida de vasos desechables en México. Disponible en https://www.reciclaunicel. com.mx/media/1111/aniqinformeejecutivojunio2013.pdf

Comisión Europea (2003). Comprender los gases efecto invernadero. Disponible en http:// ec.europa.eu/clima/sites/campaign/pdf/gases_ es.pdf 
Crutzen, P. y Stoermer, E. (2000). The "Anthropocene". Global Change Newsletter. International Geosphere-Biosphere Programme (IGBP) 41, 17-18.

Dangelico, R. M., y Pujari, D. (2010). Mainstreaming green product innovation: Why and how companies integrate environmental sustainability. Journal of Business Ethics, 95(3), 471-486, doi: https://doi.org/10.1007/s10551-010-0434-0

Dávila, F. y Varela, D. (2014). Determinación de la huella de carbono en la Universidad Politécnica Salesiana, Sede Quito, Campus Sur. Tesis de pregrado. Disponible en http://dspace.ups.edu. ec/handle/123456789/6320

Downie, J. y Stubbs, W. (2013). Evaluation of Australian companies' scope 3 greenhouse gas emissions assessments. Journal of Cleaner Production. 56, 156-163. doi: 10.1016/j. jclepro.2011.09.010

Duarte, C., Alonso, S., Benito, G., Dachs, J., Montes, C., Pardo Buendía, M. y Valladares, F. (2006). Cambio global: impacto de la actividad humana sobre el sistema tierra. Consejo Superior de Investigaciones Científicas (CSIC). Madrid, España. Disponible en http://www.csic.http:// ocw.um.es/ciencias/avances-ecologicos-parala-sostenibilidad-de-los/lectura-obligatoria-1/ leccion13/duarte-et-al-2009.pdf

Etzkowitz, H. (1998). The norms of entrepreneurial science: Cognitive effects of the new universityindustry linkages. Res. Policy, 27(8), 823-833, doi: https://doi.org/10.1016/S0048-7333(98)00093-6

García, C., Barrera, X., Gómez, R. y Suárez R. (2015). $E I A B C$ de los compromisos de Colombia para la COP 21. 2. $\mathrm{a}$ ed. WWF-Colombia. Disponible en http://www.minambiente.gov.co/images/ cambioclimatico/pdf/colombia_hacia_la_cop21/ ABC_de_los_Compromisos_de_Colombia_para_ la_cop21_VF_definitiva.pdf

Güereca, L., Torres, N. y Noyola, A. (2013). Carbon footprint as a basis for a cleaner research institute in Mexico. Journal of Cleaner Production, 47, 396403.

Gómez, N., Cadarso, M. Á., y Monsalve, F. (2016). Carbon footprint of a university in a multiregional model: the case of the University of CastillaLa Mancha. Journal of Cleaner Production, 138, 119-130, doi: https://doi.org/10.1016/j. jclepro.2016.06.009

Hannah, L. (2015). The Climate System and Climate Change. En Climate Change Biology (2. ed.). Disponible en https://doi.org/10.1016/B978-012-420218-4.00002-0

Huang, Y., Weber, C., y Matthews, S. (2009). Categorization of scope 3 emissions for streamlined enterprise carbon footprinting. Environmental Science \& Technology, 43(22), 8509-8515.

IDEAM, PNUD, MADS, DNP y Cancillería (2015). Inventario nacional de gases efecto invernadero (GEI) de Colombia. Presentado en Tercera Comunicación Nacional de Cambio Climático de Colombia. Bogotá. Colombia.

IPCC. (2007). Cambio climático 2007: informe de síntesis. Contribución de los grupos de trabajo I, II y III al Cuarto informe de evaluación del Grupo Intergubernamental de Expertos sobre el cambio climático. Disponible en https://www.ipcc.ch/pdf/ assessment-report/ar4/syr/ar4_syr_sp.pdf

IPCC. (2014). Summary for policymakers. En Climate Change 2014: Synthesis Report. Contribution of Working Groups I, II and III to the Fifth Assessment Report of the Intergovernmental Panel on Climate Change. Disponible en https:// doi.org/10.1017/сво9781107415324

Isagén (2016). Generación de energía. Disponible en https://www.isagen.com.co/SitioWeb/html/ informe-de-gestion/2016/pdf/Generacion_ energia.pdf

ISO 14064-1/2/3. (2006). Greenhouse gases: parts 1 Specification with guidance at the 
organization level for quantification and reporting of greenhouse gas emissions and removals.

Disponible en https://www.iso.org/obp/

ui\#tiso:std:iso:14064:-1:ed-1:v1:es

Isham, J., Del Negro, L. y Bission, C. (2003). Carbon neutrality at Middlebury College: A compilation of potential objectives and strategies to minimize campus climate impact. Draft prepared for the carbon reduction initiative at Middlebury College. 185 p. Disponible en http://www.middlebury. edu/system/files/media/CN\%20at\%20 MiddObjectivesandStrategies\%202003.pdf

Jorge, J. y Busquets, P. (2003). Aproximación a la huella ecológica de la Escuela Universitaria Politécnica de Manresa. España. 10p. Disponible en https://www.researchgate.net/ publication/268008202_APROXIMACION_A_ LA_HUELLA_ECOLOGICA_DE_LA_ESCUELA_ UNIVERSITARIA_POLITECNICA_DE_MANRESA_UPC

Larsen, H., Pettersen, J., Solli, C. y Hertwich, E. (2013). Investigating the carbon footprint of a University: The case of NTNU. Journal of Cleaner Production, 48, 39-47.

Lee, D., Fahey, D., Forster, P., Newton, P., Wit, R., Lim, L., Owen, B. y Sausen, R. (2009). Aviation and global climate change in the $21^{\text {st }}$ century. Atmospheric Environment, 43 (22-23), 3520-3537. DoI:10.1016/j.atmosenv.2009.04.024.

Li, X., Tan, H. y Rackes, A. (2015). Carbon footprint analysis of student behavior for a sustainable university campus in China. Journal of Cleaner Production, 106, 97-108.

Mercader, M., Ramírez de Arellano, A. y Olivares, M. (2012). Modelo de cuantificación de las emisiones de $\mathrm{CO}_{2}$ producidas en edificación derivadas de los recursos materiales consumidos en su ejecución. Informes de la Construcción, 64(527), 401-414.

Ministerio de Agricultura y Pesca, Alimentación y Medio Ambiente. (2017) ¿Qué es el cambio climático y cómo nos afecta? Disponible en http://www.mapama.gob.es/es/cambio- climatico/temas/que-es-el-cambio-climatico-ycomo-nos-afecta/

Ministerio de Educación Nacional (2016). Educación en Colombia. Disponible en http://www. mineducacion.gov.co/1759/articles-356787_ recurso_1.pdf

Ministerio de Energía, Turismo y Agenda Digital (2016). Informe Anual 2016, análisis y planes de actuación. Información sobre el gas natural. Disponible en http://www.minetad.gob.es/ energia/gas/Gas/Paginas/gasnatural.aspx

Mondéjar, M., Viñoles, R., Bastante, M., Collado, D. y Capuz, S. (2011). La huella de carbono y su utilización en las instituciones universitarias. Ponencia presentada en el XV Congreso Internacional de Ingeniería de Proyectos. Valencia, España.

Myhre, G., B.H. Samset, M. Schulz, Y. Balkanski, S. Bauer, T.K. et al. (2013). Radiative forcing of the direct aerosol effect from AeroCom Phase II simulations. Atmos. Chem. Phys., 13, 1853-1877, doi: http://doi:10.5194/acp-13-1853-2013.

National Aeronautics and Space Administration. (2017). Climate change: How do we know? Disponible en http://climate.nasa.gov/ evidence/

Robinson, O. J., Tewkesbury, A. Kemp, S. y Williams, I. (2017). Towards a universal carbon footprint standard: A case study of carbon management at universities. Journal of Cleaner Production, in press, doi: http://dx.doi.org/10.1016/j. jclepro.2017.02.147

Rodas, S. (2014). Estimación y gestión de la huella de carbono del campus central de la Universidad Rafael Landívar (tesis de pregrado). Universidad Rafael Landívar, Guatemala.

Ozawa-Meida, L., Brockway, P., Letten, K., Davies, J. y Fleming, P. (2013). Measuring carbon performance in a UK University through a consumption-based carbon footprint: de Montfort University case study. Journal of 
Cleaner Production, 56,185-198, doi: 10.1016/j. jclepro.2011.09.028.

Parga, M., y Romero, R. (2013). Ecología: impacto de la problemática ambiental actual sobre la salud y el ambiente. Colombia: Ecoe Ediciones.

Secretaría de Movilidad de Bogotá D. C. (2015). Estudio movilidad en cifras 2015. Disponible en http://www.movilidadbogota.gov.co/web/sIMUR/ ARCHIVOS/Movilidad_Cifras_2015_V4_marzo2017.pdf.

Schneider, H. y Samaniego, J. (2009). La huella del carbono en la producción, distribución y consumo de bienes y servicios. Santiago de Chile: Comisión Económica para América Latina y el Caribe (Cepal).

Sistema de Información Eléctrico Colombia (s. f.). Descripción del sistema eléctrico colombiano. Disponible en http://www.xm.com.co/Paginas/ Mercado-de-energia/descripcion-del-sistemaelectrico-colombiano.aspx

Toro, A., Gomera, A., Aguilar, J., Guijarro, C., Antúnez, M. y Vaquero, M. (2015). La huella de carbono de la Universidad de Córdoba 2015. España: Servicio de Protección Ambiental, Dirección General de Prevención y Protección Ambiental, Vicerrectorado de Coordinación Institucional e Infraestructuras, Universidad de Córdoba.
Unidad de Planeación Minero Energética (2016). Factores de emisión de combustibles colombianos. Disponible en http://www.upme. gov.co/Calculadora_Emisiones/aplicacion/ calculadora.html

Utaraskul, T. (2015). Carbon footprint of environmental science students in Suan Sunandha Rajabhat University, Thailand. Procedia-Social and Behavioral Sciences, 197, 1156-1160.

World Resources Institute y The World Business Council for Sustainable Development (WRI y WBCSD) (2013). The greenhouse gas protocol (GHG Protocol). Disponible en http://www.ghgprotocol. org/sites/default/files/ghgp/standards/ghgprotocol-revised.pdf

World Resources Institute y World Business Council for Sustainable Development (WRI y WBCSD) (2013). Technical guidance for calculating scope 3 emissions. Supplement to the corporate value chain (scope 3 ) accounting \& reporting standard. Disponible en http://www.ghgprotocol.org/ sites/default/files/ghgp/standards/Scope3_ Calculation_Guidance_0.pdf 